\title{
Modified Medical Research Council Dyspnea Scale in GOLD Classification Better Reflects Physical Activities of Daily Living
}

\author{
Anelise B Munari PT, Aline A Gulart MSc, Karoliny dos Santos MSc, Raysa S Venâncio PT, \\ Manuela Karloh PhD, and Anamaria F Mayer PhD
}

BACKGROUND: In multidimensional Global Initiative for Chronic Obstructive Lung Disease (GOLD) classification, the choice of the symptom assessment instrument (modified Medical Research Council dyspnea scale [mMRC] or COPD assessment test [CAT]) can lead to a different distribution of patients in each quadrant. Considering that physical activities of daily living (PADL) is an important functional outcome in COPD, the objective of this study was to determine which symptom assessment instrument is more strongly associated with and differentiates better the PADL of patients with COPD. METHODS: The study included 115 subjects with COPD (GOLD 2-4), who were submitted to spirometry, the MMRC, the CAT, and monitoring of PADL (triaxial accelerometer). Subjects were divided into 2 groups using the cutoffs proposed by the multidimensional GOLD classification: $\mathrm{mMRC}<2$ and $\geq 2$ and CAT $<10$ and $\geq 10$. RESULTS: Both $\mathrm{mMRC}$ and CAT reflected the PADL of COPD subjects. Subjects with mMRC $<2$ and CAT $<10$ spent less time in physical activities $<1.5$ metabolic equivalents of task (METs) (mean of the difference [95\% $\mathrm{CI}]=-62.9[-94.4$ to -31.4$], P<.001$ vs $-71.0[-116$ to -25.9$], P=.002)$ and had a higher number of steps $(3,076[1,999-4,153], P<.001$ vs $2,688[1,042-4,333], P=.002)$ than subjects with mMRC $>2$ and CAT $>10$, respectively. Physical activities $\geq 3$ METs differed only between mMRC $<2$ and mMRC $\geq 2(39.2[18.8-59.6], P<.001)$. Furthermore, only the mMRC was able to predict the PADL alone (time active, $\mathrm{r}^{2}=0.16$; time sedentary, $\mathrm{r}^{2}=0.12$; time $\geq 3 \mathrm{METs}, \mathrm{r}^{2}=0.12$ ) and associated with lung function (number of steps, $r^{2}=0.35$; walking time, $r^{2}=0.37$; time $<1.5$ METs, $r^{2}=0.25$ ). CONCLUSIONS: The mMRC should be adopted as the classification criterion for symptom assessment in the GOLD ABCD system when focusing on PADL. Key words: activities of daily living; exercise; sedentary lifestyle; symptom assessment; dyspnea; chronic obstructive pulmonary disease; GOLD classification. [Respir Care 2018;63(1):77-85. () 2018 Daedalus Enterprises]

\section{Introduction}

COPD is characterized by chronic and progressive air flow obstruction and several significant systemic manifestations that may result in reduced functional capacity and

\footnotetext{
The authors are affiliated with the Núcleo de Assistência, Ensino e Pesquisa em Reabilitação Pulmonar, Universidade do Estado de Santa Catarina (UDESC), Florianópolis, Santa Catarina, Brazil and the Programa de Pós-Graduação em Fisioterapia, Centro de Ciências da Saúde e do Esporte (CEFID), Universidade do Estado de Santa Catarina (UDESC), Florianópolis, Santa Catarina, Brazil.
}

The authors have disclosed no conflicts of interest. health status. ${ }^{1,2}$ Because of the diverse manifestations of this disease, the Global Initiative for Chronic Obstructive Lung Disease (GOLD) proposed in 2011 a multidimensional assessment (GOLD ABCD) of patients based on the severity of air flow obstruction, in addition to the unidimensional classification (GOLD I/II/III/IV). ${ }^{3}$ The risk of

\footnotetext{
Correspondence: Anamaria Fleig Mayer PhD, Physiotherapy Department; Núcleo de Assistência, Ensino e Pesquisa em Reabilitação Pulmonar, Universidade do Estado de Santa Catarina (UDESC), Rua Pascoal Simone, 358, 88080-350, Florianópolis, Brazil. E-mail: anamaria.mayer@udesc.br.
}

DOI: $10.4187 /$ respcare.05636 
future exacerbation, assessed by pulmonary function or history of exacerbations, and the symptoms, assessed by the COPD assessment test (CAT) questionnaire or the modified Medical Research Council dyspnea scale (mMRC), were used for classification. This classification system has been recently refined, and the recommendation is that the multidimensional assessment must take into account only the history of exacerbations and the evaluation of symptoms. ${ }^{4}$

The relationship between the multidimensional GOLD classification and physical activities in daily life (PADL) has been investigated in some studies. However, results are still controversial, probably because of the large number of framing possibilities in the former classification model. After the new recommendation, part of this difficulty seems to have been remedied because, from now on, the choice for the symptom assessment instrument (CAT or mMRC) represents the only aspect that may cause differences in the multidimensional classification.

Although GOLD states that it is not necessary to use more than one symptom assessment instrument to classify patients, the mMRC and CAT have been observed to have a moderate agreement. $5,6 \mathrm{Zogg}$ et $\mathrm{al}^{5}$ used the 2 symptom assessment instruments and found that the quadrants defined with the use of the mMRC correlated more strongly with the number of steps than did the quadrants established by CAT. Demeyer et $\mathrm{al}^{6}$ also suggested that the mMRC should be used along with risk assessment to better differentiate the PADL of patients with COPD. On the other hand, Moreira et $\mathrm{al}^{7}$ used the mMRC to establish the multidimensional GOLD classification and found that this classification was weakly associated with the PADL of patients with COPD.

PADL level is an important functional outcome in COPD because of its relation with the risk of exacerbations, hospitalizations, and mortality. ${ }^{8}$ However, because the symptom assessment instrument (mMRC or CAT) chosen can present different distribution of patients in the quadrants of the multidimensional classification, it is not clear whether the mMRC or CAT reflects their functional status in distinct ways. Therefore, the aim of the present study was to determine which symptom assessment instrument differentiates better the PADL of subjects with COPD and which is most strongly associated with this outcome.

\section{Methods}

\section{Participants}

Participants of the study were subjects with COPD referred to the Center of Assistance, Teaching, and Research in Pulmonary Rehabilitation (NuReab), and the recruitment occurred from March 2013 to August 2016. The inclusion criteria were: clinical diagnosis of COPD with

\section{QUICK LOOK}

\section{Current knowledge}

In the multidimensional Global Initiative for Chronic Obstructive Lung Disease (GOLD) classification, 2 instruments can be used for symptom evaluation. The choice of instrument (modified Medical Research Council dyspnea scale [mMRC] or COPD assessment test [CAT]) can lead to a different categorization of patients in each quadrant.

\section{What this paper contributes to our knowledge}

The symptom assessment instrument used in the multidimensional GOLD classification can cause differences in the distribution of patients between the $\mathrm{ABCD}$ quadrants and also in the potential to reflect their physical activity of daily living. The mMRC must be used instead of the CAT when the goal is to better discriminate the physical activity of daily living, including the sedentary behavior.

spirometric classification II-IV9, age $\geq 40 \mathrm{y}$, and clinical stability in the last month preceding the beginning of the protocol. The study excluded active smokers, patients with COPD exacerbation during the study protocol, and patients with other respiratory, cardiovascular, neurological, musculoskeletal, and rheumatologic diseases that could influence the execution of the assessments proposed.

This study was approved by the Ethics Committee on Human Research of the University of the State of Santa Catarina - Florianópolis/SC, Brazil (CAAE: 38765814.7. 0000.0118). All participants signed an informed consent form.

\section{Sample Size}

The sample size was calculated based on data from a pilot study with 20 subjects ( 14 men; $65 \pm 6$ y; $54.4 \pm 35.6$ pack-years; percent-of-predicted $\mathrm{FEV}_{1}=37.5 \pm 15.1 \%$; body mass index $=26.2 \pm 4.49 \mathrm{~kg} / \mathrm{m}^{2}$ ), using the software $\mathrm{G}^{*}$ Power 3.1.9.2. We use the mean of the difference and the highest SD of the number of steps and the walking time among subjects classified with $\mathrm{mMRC}<2$ and $\geq 2$ $(4,493 \pm 3,328$ steps and $53.8 \pm 36.8 \mathrm{~min})$ and CAT $<10$ and $\geq 10(1,996 \pm 3,416$ steps and $18.8 \pm 40.8 \mathrm{~min})$. Considering the estimation power of $80 \%$ and $\alpha$ of .05 , a maximum sample size of 104 subjects was found. In addition, to obtain a reliable measure of the number of steps $(0.80<$ intraclass correlation coefficient $>0.85)$ on 2 days of monitoring of the ADL, a sample size of approximately 100 subjects is required. ${ }^{10}$ 


\section{Protocol}

This was a cross-sectional study with protocol carried out in $3 \mathrm{~d}$. Subjects were submitted to lung function assessment, the mMRC, and the CAT questionnaire and to the monitoring of PADL.

\section{Pulmonary Function}

Pulmonary function was assessed with a portable EasyOne spirometer (ndd Medical Technologies, Zurich, Switzerland), whose calibration was checked before each assessment, following the methods and criteria recommended by the American Thoracic Society/European Respiratory Society. ${ }^{11}$ Spirometric measurements were obtained before inhalation of $400 \mu \mathrm{g}$ of bronchodilator and $15 \mathrm{~min}$ after this. Equations proposed for the Brazilian population were used for calculation of predicted values. ${ }^{12}$

\section{Symptoms}

Subjects were divided into 2 groups for analysis using the cutoffs proposed by the multidimensional GOLD classification ${ }^{1}$ : subjects with $\mathrm{mMRC}<2$ and $\mathrm{mMRC} \geq 2$ and those with $\mathrm{CAT}<10$ and $\mathrm{CAT} \geq 10$.

\section{PADL}

To evaluate the PADL, we used a triaxial accelerometer (DynaPort activity monitor, McRoberts BV, Hague, Netherlands). ${ }^{13}$ Monitoring took place on 2 consecutive weekdays, lasting $12 \mathrm{~h}$ from awakening. The mean of both days was considered for data analysis. In a previous study, 2 days of assessment were considered necessary to achieve a reliable measure $(0.70<$ intraclass reliability coefficient $<0.88) .{ }^{14}$ All participants received an explanatory manual and were instructed on how to use the equipment and register the exact time of placement and removal. Data processing and analysis were performed with the MiRA2 software (McRoberts BV, Hague, Netherlands). In cases of error of measurement after data processing and analysis, the subject used the equipment again. The following variables were considered: time spent standing, sitting, lying, and walking; movement intensity during walking; energy expenditure in PADL; and number of steps.

The sum of the time spent standing and walking corresponded to the active time, and the sum of the time spent sitting and lying represented the sedentary time. The time spent with sedentary behavior was also evaluated, considering physical activities with energy expenditure $<1.5$ metabolic equivalents of task (METs). ${ }^{15}$ In this case, a time of $\geq 8.5 \mathrm{~h}$ corresponds to inactivity. ${ }^{16}$

The time spent in moderate and vigorous physical activity ( $\geq 3$ METs), with a cutoff point of $80 \mathrm{~min} / \mathrm{d}$, was used to categorize subjects as to their level of physical (in)activity. Subjects were considered either active (physical activities $\geq 80 \mathrm{~min} / \mathrm{d}$ ) or inactive (physical activities $<80 \mathrm{~min} / \mathrm{d}$ ). ${ }^{17}$ The number of steps was used to categorize severe physical inactivity $(<4,580$ steps $) .{ }^{18}$

\section{Statistical Analysis}

Data were processed in the SPSS 20.0 (SPSS, Chicago, Illinois) and GraphPad Prism 5 (GraphPad Software, La Jolla, California) software. Data distribution was tested using the Kolmogorov-Smirnov test. The Chi-square test was used to check associations between the level of PADL and the mMRC groups $<2$ or $\geq 2$ and CAT $<10$ or $\geq 10$. The Cramer V coefficient demonstrated the strength of these associations. Simple and multiple linear regressions using the stepwise method were applied. The CAT, mMRC, and FEV 1 (percent of predicted) were considered as dependent variables, and the PADL was considered an independent variable. Correlations between CAT, mMRC, and PADL were tested using the Pearson or Spearman correlation coefficient. The intraclass correlation coefficient between days 1 and 2 of the ADL monitoring was calculated. The level of significance adopted was $P<.05$.

\section{Results}

One hundred twenty-five subjects were recruited for the study, and 115 were potentially eligible. Five of these were excluded; 3 for not meeting the spirometric criteria for diagnosis of COPD and 2 for exacerbation of the disease during the protocol. Thus, 110 subjects $(75 \mathrm{men}$; $68.2 \%$ ) completed the study. Anthropometric data, pulmonary function, PADL, dyspnea, and health status are shown in Table 1. The intraclass correlation coefficient for the PADL variables was $>0.80$.

\section{ADL Between the mMRC Cutoff 2 and CAT Cutoff 10}

Fifty-one subjects presented mMRC $<2$ (GOLD A and C), whereas 57 subjects had mMRC $\geq 2$ (GOLD B and D). Subjects with mMRC $<2$ spent less time sitting, sedentary, and in physical activities $<1.5$ METs (mean of the difference $[95 \% \mathrm{CI}]=-50.7 \mathrm{~min}[-90.4$ to $-11.4 \mathrm{~min}]$ $P=.01,-62.2 \min [-99.8$ to $-24.5 \mathrm{~min}] P=.002$, and $-62.9 \mathrm{~min}$ [ -94.4 to $-31.4 \mathrm{~min}] P<.001$, respectively) and had a higher number of steps and time standing, walking, active, and in physical activities $\geq 3$ METs (mean of the difference $[95 \% \mathrm{CI}]=3,076[1,999-4,153] P<.001$, $25.7 \mathrm{~min}[2.12-49.3 \mathrm{~min}] P=.033,35.0 \mathrm{~min}[22.3-$ $47.8 \mathrm{~min}] P<.001,70.8 \mathrm{~min}$ [35.5-106 min] $P<.001$, and $39.2 \mathrm{~min}$ [18.8-59.6 min] $P<.001$, respectively). There were no significant differences between groups 
Table 1. Anthropometric Characteristics, Lung Function, Functional Status, Dyspnea, and Health Status

\begin{tabular}{lc}
\hline \hline \multicolumn{1}{c}{ Variables } & Mean \pm SD \\
\hline Age, $y$ & $66 \pm 8$ \\
Weight, kg & $68.6 \pm 15.6$ \\
Height, m & $1.64 \pm 0.09$ \\
BMI, kg/m ${ }^{2}$ & $25.3 \pm 5.10$ \\
Smoking history, pack-years & $57.6 \pm 32.3$ \\
FEV $_{1}$, L & $1.00 \pm 0.47$ \\
FEV $_{1}, \%$ predicted & $35.1 \pm 15.6$ \\
FVC, L & $2.24 \pm 0.71$ \\
FVC, $\%$ predicted & $60.8 \pm 17.2$ \\
FEV $/$ FVC & $0.44 \pm 0.11$ \\
Steps, no./d & $5,292 \pm 3,200$ \\
Time walking, min & $67.4 \pm 31.5$ \\
Time standing, min & $149 \pm 62.4$ \\
Time sitting, min & $378 \pm 105$ \\
Time lying, min & $101 \pm 87.2$ \\
Movement intensity, m/s ${ }^{2}$ & $1.72 \pm 0.27$ \\
Total energy expenditure, kcal & $1,264 \pm 298$ \\
mMRC* & $2(0-4)$ \\
CAT total & $18 \pm 8$ \\
& \\
* Median (minimum-maximum). & \\
BMI = body mass index & \\
mMRC = modified Medical Research Council dyspnea scale & \\
CAT $=$ COPD assessment test & \\
\hline
\end{tabular}

(mean of the difference $[95 \% \mathrm{CI}]=-11.5 \min [-44.5$ to $21.5 \mathrm{~min}], P=.300$ ) with respect to the lying time.

Sixteen subjects presented CAT $<10$ (GOLD A and C), whereas 94 subjects presented CAT $\geq 10$ (GOLD B and D). Subjects with CAT $<10$ spent less time in physical activities $<1.5$ METs (mean of the difference $[95 \% \mathrm{CI}]=$ $-71.0 \mathrm{~min}[-116$ to $-25.9 \mathrm{~min}], P=.002)$ and had a higher number of steps and time walking and active (mean of the difference $[95 \% \mathrm{CI}]=2,688[1,042-4,333] P=$ $.002,33.0 \mathrm{~min}[13.8-52.2 \mathrm{~min}] P=.002$, and $59.3 \mathrm{~min}$ [7.45-111 min] $P=.036$, respectively). Time sitting, lying, standing, and in physical activities $\geq 3$ METs were similar between the 2 groups (mean of the difference $[95 \% \mathrm{CI}]=-50.3 \mathrm{~min}[-107$ to $5.92 \mathrm{~min}] P=.08,-4.39$ [ -51.3 to $42.6 \mathrm{~min}] P=.83,17.9 \mathrm{~min}[-15.5$ to $51.4 \mathrm{~min}$ ] $P=.34$, and $15.9 \mathrm{~min}$ [ -14.3 to $46.0 \mathrm{~min}$ ] $P=.08$, respectively). Figure 1 shows the main results of comparisons between mMRC $<2$ and $\geq 2$ (A) and between $\mathrm{CAT}<10$ and $\geq 10$ (B).

Both classifications, the ones based on cutoff of 2 for mMRC and 10 for CAT, were associated with the classification based on the cutoff of $80 \mathrm{~min}$ in physical activities $\geq 3$ METs, with the sedentarism classification based on the cutoff point of $8.5 \mathrm{~h}$ in physical activities $<1.5$ METs and with the severe physical inactivity based on the cutoff of $4,580 \mathrm{steps} / \mathrm{d}$. Details of results of the associations are listed in Table 2.
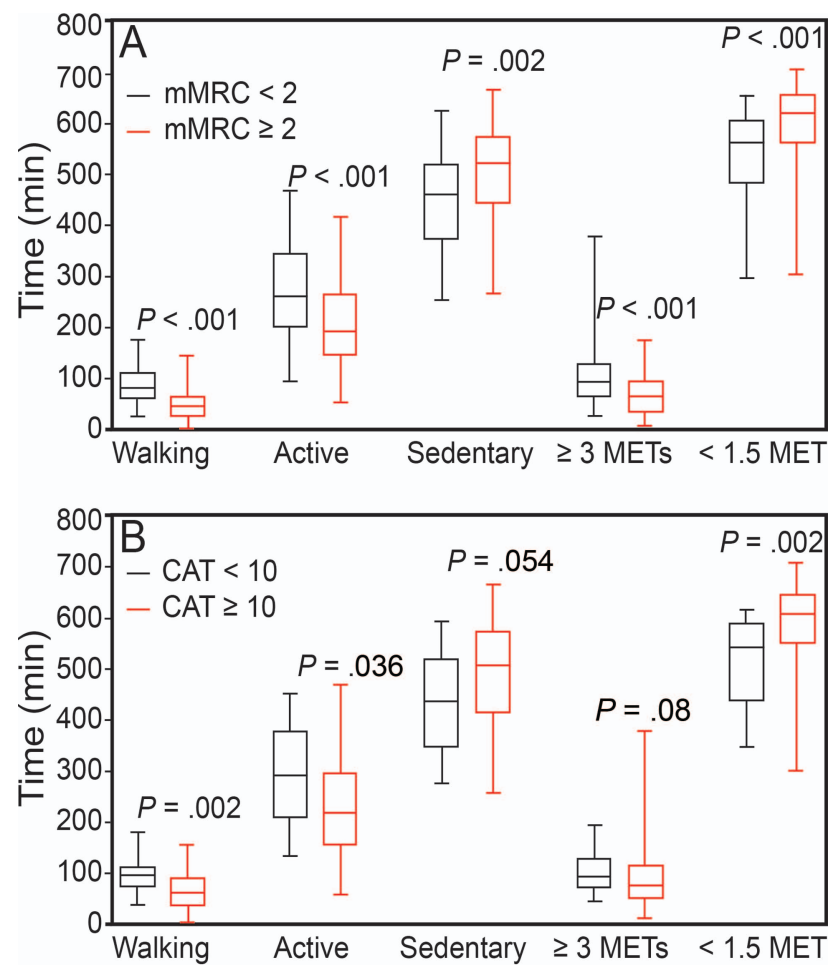

Fig. 1. Comparisons of time walking, active time, sedentary time, time in physical activity $<1.5$ metabolic equivalents of task (METs), and time in physical activity $\geq 3$ METs between Modified Medical Research Council dyspnea scale (mMRC) (A) and COPD assessment test (CAT) (B). Center lines represent the median; the top and bottom lines (box) represent interquartile range; and top and bottom whiskers represent quartile $3+1.5$ (quartile 3 - quartile 1) and quartile $1-1.5$ (quartile 3 - quartile 1 ), respectively.

\section{Correlations Between Physical Activity in Daily Life and Dyspnea, Health Status, and Pulmonary Function}

The mMRC generally showed stronger correlation with PADL than CAT. The results of the correlations between PADL variables and $\mathrm{mMRC}, \mathrm{CAT}$, and $\mathrm{FEV}_{1}$ (in liters and percent predicted) are described in Table 3 .

\section{Simple Linear Regression and Predictive Models for ADL}

The variability of $\mathrm{FEV}_{1}$ percent predicted, mMRC, and CAT were able to explain, in isolation, $23(P<.001), 29$ $(P<.001)$, and $17 \%(P<.001)$ of the variability in the number of steps, respectively; $26(P<.001), 28(P<.001)$, and $17 \%(P<.001)$ of the variability of the time walking; $8(P=.002), 16(P<.001)$, and 8\% $(P=.003)$ of the variability of active time; $7(P=.006), 12(P<.001)$, and $7 \%(P=.007)$ of the variability of sedentary time; and 21 $(P<.001), 17(P<.001)$, and $11 \%(P<.001)$ of the variability of time in physical activities $<1.5$ METs, re- 
Table 2. Distribution of Subjects' Physical Activity in Daily Life Outcomes and Association With the Modified Medical Research Council Dyspnea Scale Cutoff 2 and COPD Assessment Test Cutoff 10

\begin{tabular}{|c|c|c|c|c|}
\hline \multirow{2}{*}{ Outcomes } & \multicolumn{4}{|c|}{ mMRC Cutoff 2} \\
\hline & $\operatorname{mMRC}<2(n=51)$ & $\operatorname{mMRC} \geq 2(n=57)$ & Cramer's V & $P$ \\
\hline \multicolumn{5}{|l|}{ Time in PADL $\geq 3$ METs } \\
\hline$\geq 80 \min$ (active) & $37(72.5 \%)$ & $15(26.3 \%)$ & 0.46 & $<.001$ \\
\hline$<80 \min$ (inactive) & $14(27.5 \%)$ & $42(73.7 \%)$ & & \\
\hline \multicolumn{5}{|l|}{ Time in PADL $<1.5$ METs } \\
\hline$\geq 510$ min (sedentary) & $37(72.5 \%)$ & $6(10.5 \%)$ & 0.22 & .02 \\
\hline$<510 \min ($ not sedentary) & $14(27.5 \%)$ & $51(89.5 \%)$ & & \\
\hline \multicolumn{5}{|l|}{ Steps per day } \\
\hline$\geq 4,580 \mathrm{steps} / \mathrm{d}$ (not severe inactivity) & $40(78.4 \%)$ & $18(31.6 \%)$ & 0.47 & $<.001$ \\
\hline \multirow[t]{3}{*}{$<4,580$ steps/d (severe inactivity) } & $11(21.6 \%)$ & $39(68.4 \%)$ & & \\
\hline & \multicolumn{4}{|c|}{ CAT Cutoff 10} \\
\hline & CAT < $10(n=16)$ & $\mathrm{CAT} \geq 10(n=94)$ & Cramer's V & $P$ \\
\hline \multicolumn{5}{|l|}{ Time in PADL $\geq 3$ METs } \\
\hline$\geq 80 \min$ (active) & $13(81.2 \%)$ & $40(42.6 \%)$ & 0.27 & .004 \\
\hline$<80 \min$ (inactive) & $3(18.8 \%)$ & $54(57.4 \%)$ & & \\
\hline \multicolumn{5}{|l|}{ Time in PADL $<1.5$ METs } \\
\hline$\geq 510$ min (sedentary) & $10(62.5 \%)$ & $14(14.9 \%)$ & 0.21 & .030 \\
\hline$<510 \min$ (not sedentary) & $6(37.5 \%)$ & $80(85.1 \%)$ & & \\
\hline \multicolumn{5}{|l|}{ Steps per day } \\
\hline$\geq 4,580$ steps/d (not severe inactivity) & $13(81.2 \%)$ & $46(48.9 \%)$ & 0.23 & .02 \\
\hline$<4,580$ steps/d (severe inactivity) & $3(18.8 \%)$ & $48(51.1 \%)$ & & \\
\hline \multicolumn{5}{|c|}{$\begin{array}{l}\text { Results are } n(\%) \\
\text { PADL }=\text { physical activity in daily life } \\
\text { mMRC = Modified Medical Research Council dyspnea scale } \\
\text { MET }=\text { metabolic equivalent of task } \\
\text { CAT }=\text { COPD assessment test }\end{array}$} \\
\hline
\end{tabular}

Table 3. Correlation Coefficient Between Physical Activity in Daily Life Variables and Dyspnea, Health Status, and Pulmonary Function

\begin{tabular}{|c|c|c|c|c|}
\hline \multirow[b]{2}{*}{ Characteristics } & \multicolumn{4}{|c|}{$\mathrm{r}$} \\
\hline & mMRC & CAT & $\begin{array}{l}\mathrm{FEV}_{1} \\
(\mathrm{~L})\end{array}$ & $\begin{array}{c}\mathrm{FEV}_{1} \\
(\% \text { Predicted })\end{array}$ \\
\hline Steps (no./d) & $-0.58 *$ & $-0.38 *$ & $0.52 *$ & $0.55^{*}$ \\
\hline Time walking (min) & $-0.57 *$ & $-0.37 *$ & $0.52 *$ & $0.55^{*}$ \\
\hline Time standing (min) & $-0.22 *$ & -0.06 & -0.03 & 0.07 \\
\hline Time sitting (min) & $0.29 *$ & $0.20 *$ & $-0.20 *$ & $-0.21 *$ \\
\hline Time lying (min) & 0.09 & 0.03 & -0.02 & -0.08 \\
\hline Time $\geq 3$ METs & $-0.37 *$ & $-0.29 *$ & $0.29 *$ & $-0.47 *$ \\
\hline Time $<1.5$ METs & $0.49 *$ & 0.28 & $-0.36 *$ & $0.22 *$ \\
\hline Movement intensity $\left(\mathrm{m} / \mathrm{s}^{2}\right)$ & $-0.55^{*}$ & $-0.37 *$ & $0.42 *$ & $0.36^{*}$ \\
\hline $\mathrm{mMRC}$ & & $0.62 *$ & $-0.51 *$ & $-0.49 *$ \\
\hline CAT & & & $-0.43 *$ & $-0.35 *$ \\
\hline \multicolumn{5}{|c|}{$\begin{array}{l}* P<.05 \\
\text { mMRC }=\text { Modified Medical Research Council dyspnea scale } \\
\text { CAT }=\text { COPD assessment test } \\
\text { MET }=\text { metabolic equivalent of task }\end{array}$} \\
\hline
\end{tabular}

spectively. The variability of the time in physical activities $\geq 3$ METs was explained in $12 \%$ by mMRC $(P<.001)$ and in $5 \%$ by CAT $(P=.02)$, whereas percent-of-predicted $\mathrm{FEV}_{1}$ was not able to explain this variable $(P=.055)$.

When tested in predictive models for variables of PADL, it was observed that CAT was not retained in any of them, whereas mMRC was in all models. The results of multiple regression are presented in Table 4.

\section{Discussion}

The present study aimed to determine which symptom assessment instrument better differentiates the PADL of subjects with COPD and is most strongly associated with this outcome. The main findings demonstrate that although the CAT and mMRC are able to reflect the level of ADL of COPD subjects, the mMRC has a stronger association. Furthermore, only the mMRC was able to predict the PADL alone, and this measure was also associated with lung function.

Since the publication of the new COPD classification model (GOLD ABCD) by GOLD in 2011, noted as an 
Table 4. Model Predictor for Time Walking, Time Active, Time Sedentary, Time in Physical Activities $\geq 3$ Metabolic Equivalents of Task, and Time in Physical Activities $<1.5$ Metabolic Equivalents of Task

\begin{tabular}{|c|c|c|c|c|c|}
\hline & $\begin{array}{l}\text { Coefficient of } \\
\text { Regression }\end{array}$ & SE & $95 \% \mathrm{CI}$ & $r^{2}$ & $P$ \\
\hline \multicolumn{6}{|l|}{ Steps (no./d) } \\
\hline Constant & 5,199 & 1,004 & $3,208-7,189$ & & $<.001$ \\
\hline mMRC & -981 & 227 & $-1,432$ to -530 & & $<.001$ \\
\hline $\mathrm{FEV}_{1}(\%$ predicted $)$ & 66.7 & 20.8 & $25.4-108$ & 0.35 & .002 \\
\hline \multicolumn{6}{|l|}{ Time walking (min) } \\
\hline Constant & 61.0 & 11.5 & $38.2-83.7$ & & $<.001$ \\
\hline mMRC & -10.8 & 2.61 & -15.8 to -5.63 & & $<.001$ \\
\hline $\mathrm{FEV}_{1}(\%$ predicted $)$ & 0.82 & 0.22 & $0.39-1.25$ & 0.37 & $<.001$ \\
\hline \multicolumn{6}{|l|}{ Time active (min) } \\
\hline Constant & 299 & 16.5 & $267-332$ & & $<.001$ \\
\hline mMRC & -30.5 & 6.90 & -44.2 to -16.8 & 0.16 & $<.001$ \\
\hline \multicolumn{6}{|l|}{ Time sedentary (min) } \\
\hline Constant & 422 & 17.6 & $387-457$ & & $<.001$ \\
\hline mMRC & 27.8 & 7.34 & $13.2-42.3$ & 0.12 & $<.001$ \\
\hline \multicolumn{6}{|l|}{ Time $\geq 3$ METs (min) } \\
\hline Constant & 119 & 9.68 & $99.5-138$ & & $<.001$ \\
\hline mMRC & -15.1 & 4.04 & -23.1 to -7.10 & 0.12 & $<.001$ \\
\hline \multicolumn{6}{|l|}{ Time $<1.5$ METs (min) } \\
\hline Constant & 598 & 29.7 & $539-657$ & & $<.001$ \\
\hline $\mathrm{FEV}_{1}(\%$ predicted $)$ & -1.98 & 0.62 & -3.21 to -0.76 & & .002 \\
\hline mMRC & 17.5 & 6.74 & $4.09-30.8$ & 0.25 & .01 \\
\hline
\end{tabular}

$\overline{\mathrm{mMRC}}=$ modified Medical Research Council dyspnea scale

$\mathrm{MET}=$ metabolic equivalent of task

important advance because it incorporated multimodality assessment and symptom burden and highlighted the importance of exacerbation prevention in the management of COPD, ${ }^{1}$ a considerable number of studies have sought to analyze the equivalence of different classification criteria $^{5,19-27}$ and their association with important outcomes, such as functional status, ${ }^{5-7,28-30}$ quality of life, ${ }^{29,31,32}$ and mortality. ${ }^{33-37}$ Recently, a systematic review ${ }^{4}$ found that there is an average classification disagreement of $13 \%$ in all quadrants, depending on the instrument used. The agreement between CAT and mMRC ranged from slight to moderate, and the meta-analysis showed a pooled kappa coefficient of $0.548\left(95 \%\right.$ CI $0.35-0.70, P<.001 ; \mathrm{I}^{2}=$ 99.3; $\mathrm{z}=4.84)$. These findings indicate that CAT $\geq 10$ and $\mathrm{mMRC} \geq 2$ are not equivalent when assessing symptoms in patients with COPD. 4

In the present study, both symptom assessment instruments were associated with categorizations of PADL (physical activity, sedentarism, and severe inactivity). However, the associations of PADL and symptoms with mMRC score were stronger than with CAT. Also, whereas all variables related to PADL (except for time lying) differed among subjects with $\mathrm{mMRC}<2$ and $\mathrm{mMRC} \geq 2$, the time lying, sitting, standing, and in physical activities $\geq 3$ METs did not differ between subjects with $\mathrm{CAT}<10$ and $\mathrm{CAT} \geq 10$. These findings, added to the fact that CAT was not re- tained in any predictive model of PADL, suggest that the mMRC better reflects the performance of subjects in their activities than CAT, especially in high-energy expenditure activities ( $\geq 3$ METs). A possible explanation is that although CAT encompasses the major symptoms of patients with COPD, ${ }^{38}$ some of its items may not substantially interfere with the realization of PADL, such as cough and expectoration. In contrast, the mMRC specifically rates dyspnea from minimum to maximum physical exertion, symptoms more strongly linked to functional limitations in patients with COPD. ${ }^{39}$

In a previous study, ${ }^{40}$ the $\mathrm{FEV}_{1}$ did not show a correlation with certain ADL variables, differing from the findings of the present study, which showed moderate correlations with steps and time walking. Furthermore, in isolation, mMRC and $\mathrm{FEV}_{1}$ were able to predict a large part of PADL variables and, when combined, explained more strongly the number of steps, the time walking, and the time in physical activities $<1.5$ METs. Therefore, although $\mathrm{FEV}_{1}$ alone does not reflect $\mathrm{ADL}$ in patients with COPD as well, it may be possible to achieve a more complete analysis of this outcome when $\mathrm{FEV}_{1}$ is associated with a symptom scale, as was the case in the previous GOLD ABCD classification based on the mMRC.

To our knowledge, only 3 studies investigating the functional status in the multidimensional GOLD classification 
have objectively ascertained the differences in PADL between the ABCD quadrants. ${ }^{5-7}$ In a study developed by Zogg et $\mathrm{al}^{5}$ among the PADL variables (number of steps, active energy expenditure, level of physical activities, and time in physical activities $>3$ METs), only the number of steps differed between quadrants, regardless of the use of CAT or mMRC. However, mMRC correlated more strongly with the number of steps than CAT ( $\mathrm{r}=-0.51$ vs $\mathrm{r}=$ $-0.37, P<.001$ in both cases, respectively). Moreira et $\mathrm{al}^{7}$ showed that both GOLD classifications (A-D and I-IV) are weakly associated with PADL variables (Cramer's $\mathrm{V}<0.20$ for all). In addition, no differences were found between active and inactive time (physical activities $>2$ and 3 METs and physical activities $<2$ and 3 METs) between quadrants $(P=.09$ to .39$)$. More recently, Demeyer et $\mathrm{al}^{6}$ showed that the mMRC is preferable when used in combination with risk assessment to differentiate PADL of patients with COPD. Furthermore, regardless of risk assessment, the mMRC can be a good predictor of mortality, ${ }^{34,39}$ since the higher the score in mMRC, the fewer the number of steps. ${ }^{6}$

In contrast to previous studies, this study conducted a more detailed analysis of PADL, including sedentary behavior. Patients with COPD adopt sedentary behavior throughout most of the day, most frequently carrying out physical activities $<1.5$ METs in seated or reclined positions. ${ }^{15,41}$ This pattern of behavior has also been observed even when patients are considered physically active (ie, when they perform $\geq 80 \mathrm{~min}$ of moderate to vigorous physical activities per day $[\geq 3 \mathrm{METs}]) \cdot{ }^{17} \mathrm{It}$ is known that sedentary behavior is associated with negative health effects in the general population, increasing the risk of cardiovascular and metabolic diseases and mortality. ${ }^{42}$ In patients with COPD, the risk of death is about 4 times higher in those who spend $>8.5 \mathrm{~h}$ in physical activities $<1.5$ METs. ${ }^{16}$ Furthermore, for each hour of the day spent in sedentary physical activities, the risk of death increases by $42 \% .{ }^{16}$ In the present study, only the score on the mMRC correlated with the time in physical activities $<1.5$ METs, and the magnitude of the difference observed among subjects with $\mathrm{mMRC}<2$ and $\mathrm{mMRC} \geq 2$ was higher than among subjects with $\mathrm{CAT}<10$ and $\mathrm{CAT} \geq 10$. These results suggest that the mMRC reflects sedentary behavior better than CAT does.

Thus, the symptom assessment instrument used in the multidimensional GOLD classification can cause not only differences in the distribution of patients between the $\mathrm{ABCD}$ quadrants, but also in the potential to reflect their PADL. Therefore, standardizing the choice of the symptom assessment instrument can be a determining factor. This has been discussed in the literature in an analysis of 4 cohort studies. ${ }^{43}$ Although GOLD recommends the use of either one of the 2 instruments for the multidimensional classification, ${ }^{1}$ the results of the present study suggest that, sup- ported by a previous study, ${ }^{6}$ the mMRC must be used instead of the CAT when the goal is to better discriminate the PADL, including the sedentary behavior. It is important to consider this outcome while evaluating patients with COPD, since sedentary behavior has a causal relationship with mortality in the general population ${ }^{44}$ and also in these patients. ${ }^{16}$

The heterogeneous distribution of subjects in the groups formed by CAT (16 subjects with CAT $<10 ; 94$ subjects with CAT $\geq 10$ ) could be considered a limitation of this study. This may have caused a type- 2 error in some comparisons. However, the sample size in the present study exceeded the previous calculation. Furthermore, these same conditions are observed in most studies that have addressed GOLD classifications. ${ }^{5-7,24,28,29,31,34}$ The absence of GOLD I subjects in the sample of the present study prevents us from generalizing the results for these patients. However, the selection of patients in the disease's early stages is difficult because underdiagnosis is common, especially at this stage. ${ }^{1}$ In addition, GOLD I patients may be asymptomatic, and therefore the impact of the disease may be very low and clinically not significant. PADL analysis performed only in 2 consecutive days could also be considered a limitation of the present study, but both of the variables used to estimate sample size (number of steps and walking time) and sedentary behavior showed high intraclass correlation coefficient values $(>0.80)$.

To our knowledge, this was the first study to demonstrate that the symptom assessment instrument chosen for the multidimensional GOLD classification results in better differentiation of variables, reflecting physical inactivity and sedentary behavior. Furthermore, only the mMRC score, regardless of association with $\mathrm{FEV}_{1}$, was able to explain the variability of PADL in patients with COPD.

\section{Conclusions}

The multidimensional GOLD classification requires standardization regarding the criterion for symptom assessment. Although physical inactivity and sedentary lifestyles are striking features among patients in the D quadrant $(\mathrm{mMRC} \geq 2$ or CAT $\geq 10$ ), we suggest that the mMRC should be adopted as the classification criterion in the GOLD ABCD system, especially when the focus is the level of PADL.

\section{REFERENCES}

1. Global Initiative for Chronic Obstructive Lung Disease. Global strategy for the diagnosis, management, and prevention of chronic obstructive pulmonary disease. Updated 2017. http://goldcopd.org/gold2017-global-strategy-diagnosis-management-prevention-copd/.

2. Barnes PJ, Celli BR. Systemic manifestations and comorbidities of COPD. Eur Respir J 2009;33(5):1165-1185. 
3. From the Global Strategy for the Diagnosis, Management and Prevention of COPD, Global Initiative for Chronic Obstructive Lung Disease (GOLD) 2011 Available from: http://www.goldcopd.org/.

4. Karloh M, Fleig Mayer A, Maurici R, Pizzichini MM, Jones PW, Pizzichini E. The COPD assessment test: what do we know so far?: a systematic review and meta-analysis about clinical outcomes prediction and classification of patients into GOLD stages. Chest 2016; 149(2):413-425.

5. Zogg S, Dürr S, Miedinger D, Steveling EH, Maier S, Leuppi JD. Differences in classification of COPD patients into risk groups A-D: a cross-sectional study. BMC Res Notes 2014;7:562.

6. Demeyer H, Gimeno-Santos E, Rabinovich RA, Hornikx M, Louvaris $\mathrm{Z}$, de Boer WI, et al. Physical activity characteristics across GOLD quadrants depend on the questionnaire used. PLoS One 2016; 11(3): 0151255.

7. Moreira GL, Donaria L, Furlanetto KC, Paes T, Sant'Anna T, Hernandes NA, Pitta F. GOLD B-C-D groups or GOLD II-III-IV grades: which one better reflects the functionality of patients with chronic obstructive pulmonary disease? Chron Respir Dis 2015;12(2):102110.

8. Gimeno-Santos E, Frei A, Steurer-Stey C, de Batlle J, Rabinovich RA, Raste Y, et al. Determinants and outcomes of physical activity in patients with COPD: a systematic review. Thorax 2014;69(8):731739.

9. Vestbo J, Hurd SS, Agustí AG, Jones PW, Vogelmeier C, Anzueto A, et al. Global strategy for the diagnosis, management, and prevention of chronic obstructive pulmonary disease: GOLD executive summary. Am J Respir Crit Care Med 2013;187(4):347-365.

10. Demeyer H, Burtin C, Van Remoortel H, Hornikx M, Langer D, Decramer M, et al. Standardizing the analysis of physical activity in patients with COPD following a pulmonary rehabilitation program. Chest 2014;146(2):318-327.

11. Miller MR, Hankinson J, Brusasco V, Burgos F, Casaburi R, Coates A, et al. Standardisation of spirometry. Eur Respir J 2005;26(2):319338.

12. Pereira CA, Sato T, Rodrigues SC. New reference values for forced spirometry in white adults in Brazil. J Bras Pneumol 2007;33(4): 397-406.

13. Van Remoortel H, Raste Y, Louvaris Z, Giavedoni S, Burtin C, Langer D, et al. Validity of six activity monitors in chronic obstructive pulmonary disease: a comparison with indirect calorimetry. PLoS One 2012;7(6):e39198.

14. Pitta F, Troosters T, Spruit MA, Probst VS, Decramer M, Gosselink R. Characteristics of physical activities in daily life in chronic obstructive pulmonary disease. Am J Respir Crit Care Med 2005;171(9): 972-977.

15. Hill K, Gardiner PA, Cavalheri V, Jenkins SC, Healy GN. Physical activity and sedentary behaviour: applying lessons to chronic obstructive pulmonary disease. Intern Med J 2015;45(5):474-482.

16. Furlanetto KC, Donária L, Schneider LP, Lopes JR, Ribeiro M, Hernandes KB, et al. Sedentary behavior is an independent predictor of mortality in subjects with COPD. Respir Care 2017;62(5):579587.

17. van Remoortel H, Camillo CA, Langer D, Hornikx M, Demeyer H, Burtin C, et al. Moderate intense physical activity depends on selected metabolic equivalent of task (MET) cut-off and type of data analysis. PLoS One 2013;8(12):e84365.

18. Depew ZS, Novotny PJ, Benzo RP. How many steps are enough to avoid severe physical inactivity in patients with chronic obstructive pulmonary disease? Respirology 2012;17(6):1026-1027.

19. Jones PW, Nadeau G, Small M, Adamek L. Characteristics of a COPD population categorised using the GOLD framework by health status and exacerbations. Respir Med 2014;108(1):129-135.
20. Price DB, Baker CL, Zou KH, Higgins VS, Bailey JT, Pike JS. Real-world characterization and differentiation of the Global Initiative for Chronic Obstructive Lung Disease strategy classification. Int J Chron Obstruct Pulmon Dis 2014;9:551-561.

21. Han J, Dai L, Zhong N, Young D. Breathlessness or health status in chronic obstructive pulmonary disease: the impact of different definitions. COPD 2015;12(2):115-125.

22. Jones PW, Adamek L, Nadeau G, Banik N. Comparisons of health status scores with MRC grades in a primary care COPD population: implications for the new GOLD 2011 classification. Eur Respir J 2013;42(3):647-654

23. Kim S, Oh J, Kim YI, Ban HJ, Kwon YS, Oh IJ, et al. Differences in classification of COPD group using COPD assessment test (CAT) or modified Medical Research Council (mMRC) dyspnea scores: a cross-sectional analyses. BMC Pulm Med 2013(Can't get issue from record); 13:35.

24. Rieger-Reyes C, García-Tirado FJ, Rubio-Galán FJ, Marín-Trigo JM. Classification of chronic obstructive pulmonary disease severity according to the new Global Initiative for Chronic Obstructive Lung Disease 2011 guidelines: COPD assessment test versus modified Medical Research Council scale. Arch Bronconeumol 2014;50(4): 129-134.

25. Casanova C, Marin JM, Martinez-Gonzalez C, de Lucas-Ramos P, Mir-Viladrich I, Cosio B, et al. New GOLD classification: longitudinal data on group assignment. Respir Res 2014;15:3.

26. Pillai AP, Turner AM, Stockley RA. Global Initiative for Chronic Obstructive Lung Disease 2011 symptom/risk assessment in $\alpha 1$ antitrypsin deficiency. Chest 2013;144(4):1152-1162.

27. Han MK, Muellerova H, Curran-Everett D, Dransfield MT, Washko GR, Regan EA, et al. GOLD 2011 disease severity classification in COPDGene: a prospective cohort study. Lancet Respir Med 2013; 1(1):43-50.

28. García-Rio F, Soriano JB, Miravitlles M, Muñoz L, Duran-Tauleria E, Sánchez G, et al. Frequency of multi-dimensional COPD indices and relation with disease activity markers. COPD 2013;10(4):436-443.

29. Barusso MS, Gianjoppe-Santos J, Basso-Vanelli RP, Regueiro EM, Panin JC, Di Lorenzo VA. Limitation of activities of daily living and quality of life based on COPD combined classification. Respir Care 2015;60(3):388-398

30. Durheim MT, Smith PJ, Babyak MA, Mabe SK, Martinu T, WeltyWolf KE, et al. Six-minute-walk distance and accelerometry predict outcomes in chronic obstructive pulmonary disease independent of Global Initiative for Chronic Obstructive Lung Disease 2011 Group. Ann Am Thorac Soc 2015;12(3):349-356.

31. Miravitlles M, Huerta A, Fernández-Villar JA, Alcázar B, Villa G, Forné $\mathrm{C}$, et al. Generic utilities in chronic obstructive pulmonary disease patients stratified according to different staging systems. Health Qual Life Outcomes 2014;12:120.

32. Boland MR, Tsiachristas A, Kruis AL, Chavannes NH, Rutten-van Mölken MP. Are GOLD ABCD groups better associated with health status and costs than GOLD 1234 grades? A cross-sectional study. Prim Care Respir J 2014;23(1):30-37.

33. Leivseth L, Brumpton BM, Nilsen TI, Mai XM, Johnsen R, Langhammer A. GOLD classifications and mortality in chronic obstructive pulmonary disease: the HUNT Study, Norway. Thorax 2013; 68(10):914-921.

34. Casanova C, Marin JM, Martinez-Gonzalez C, de Lucas-Ramos P, Mir-Viladrich I, Cosio B, et al. Differential effect of modified medical research council dyspnea, COPD assessment test, and clinical COPD questionnaire for symptoms evaluation within the new GOLD staging and mortality in COPD. Chest 2015;148(1): 159-168.

35. Soriano JB, Lamprecht B, Ramírez AS, Martinez-Camblor P, Kaiser $\mathrm{B}$, Alfageme I, et al. Mortality prediction in chronic obstructive 


\section{MMRC IN GOLD AND Physical ADL}

pulmonary disease comparing the GOLD 2007 and 2011 staging systems: a pooled analysis of individual patient data. Lancet Respir Med 2015;3(6):443-450.

36. Lange P, Marott JL, Vestbo J, Olsen KR, Ingebrigtsen TS, Dahl M, Nordestgaard BG. Prediction of the clinical course of chronic obstructive pulmonary disease, using the new GOLD classification: a study of the general population. Am J Respir Crit Care Med 2012;186(10):975-981.

37. Agusti A, Edwards LD, Celli B, Macnee W, Calverley PM, Mullerova H, et al. Characteristics, stability and outcomes of the 2011 GOLD COPD groups in the ECLIPSE cohort. Eur Respir J 2013;42(3): 636-646.

38. Jones PW, Harding G, Berry P, Wiklund I, Chen WH, Kline Leidy N. Development and first validation of the COPD assessment test. Eur Respir J 2009;34(3):648-654.

39. Nishimura K, Izumi T, Tsukino M, Oga T. Dyspnea is a better predictor of 5-year survival than airway obstruction in patients with COPD. Chest 2002;121(5):1434-1440.
40. Pitta F, Takaki MY, Oliveira NH, Sant'anna TJ, Fontana AD, Kovelis D, et al. Relationship between pulmonary function and physical activity in daily life in patients with COPD. Respir Med 2008;102(8): 1203-1207.

41. Hunt T, Madigan S, Williams MT, Olds TS. Use of time in people with chronic obstructive pulmonary disease: a systematic review. Int J Chron Obstruct Pulmon Dis 2014;9:1377-1388.

42. Wilmot EG, Edwardson CL, Achana FA, Davies MJ, Gorely T, Gray $\mathrm{LJ}$, et al. Sedentary time in adults and the association with diabetes, cardiovascular disease and death: systematic review and meta-analysis. Diabetologia 2012;55(11):2895-2905.

43. Agusti A, Hurd S, Jones P, Fabbri LM, Martinez F, Vogelmeier C, et al. FAQs about the GOLD 2011 assessment proposal of COPD: a comparative analysis of four different cohorts. Eur Respir J 2013; 42(5):1391-1401.

44. Biddle SJ, Bennie JA, Bauman AE, Chau JY, Dunstan D, Owen N, et al. Too much sitting and all-cause mortality: is there a causal link? BMC Public Health 2016;16:635. 